\title{
Research on RBV Control Strategy of Large Angle Maneuver
}

\author{
Jiangtao $\mathrm{Xu},{ }^{1} \mathrm{Na}$ Luo, ${ }^{1} \mathrm{Yu} \mathrm{Fu}{ }^{2}$ Litong Wang, ${ }^{1}$ and Xiande $\mathrm{Wu}{ }^{1}$ \\ ${ }^{1}$ Department of Astronautics Engineering, Harbin Engineering University, Harbin 150001, China \\ ${ }^{2}$ Beijing Institute of Astronautical Systems Engineering, Beijing 100076, China \\ Correspondence should be addressed to Jiangtao Xu; hit_xjt@163.com
}

Received 11 July 2013; Revised 20 October 2013; Accepted 21 November 2013; Published 28 January 2014

Academic Editor: Lixian Zhang

Copyright (C) 2014 Jiangtao Xu et al. This is an open access article distributed under the Creative Commons Attribution License, which permits unrestricted use, distribution, and reproduction in any medium, provided the original work is properly cited.

\begin{abstract}
Considering the hypersonic aerospace vehicle, with high dynamic, strong varying parameters, strong nonlinear, strong coupling, and the complicated flight environment, conventional flight control methods based on linear system may become invalid. To the high precision and reliable control problem of this vehicle, nonlinear flight control strategy based on neural network robust adaptive dynamic inversion is proposed. Firstly, considering the nonlinear characteristics of aerodynamic coefficients varying with Mach numbers, attack angle, and sideslip angle, the complete nonlinear 6-DOF model of RBV is established. Secondly, based on the timescale separation, using the nonlinear dynamic inversion control strategy achieves the pseudolinear decoupling of RBV. And then, using the neural network with single hidden layer approximates the dynamic inversion error for system model uncertainty. Next, the external disturbance and network approximating error are suppressed by robust adaptive control. Finally, using Lyapunov's theory proves that all error signals of closed loop system are uniformly bounded finally under this control strategy. Nonlinear simulation verifies the feasibility and validity of this control strategy to the RBV control system.
\end{abstract}

\section{Introduction}

To meet the development of space research, space tourism, and military requirement and achieve the goal of speediness, high reliability, reusability, and low cost, the hypersonic aerospace vehicle especially reusable launch vehicle, RLV, emerges [1-6].

Reusable boosted vehicle (RBV) experiences the subsonic, transonic, and supersonic phase during the whole flight, which the aerodynamic coefficients, dynamic pressure, and flight altitude acute change, especially in the large attitude adjusting phase with supersonic and large angle of attack. Considering the imprecise aerodynamic model, abominable flight condition at the high altitude, great system perturbation, and interference, gain scheduling and PID control strategy based on linearized with small disturbances cannot apply to the flight control system of RBV large attitude adjusting phase design. It is necessary to design RBV control system by using nonlinear control method.

Shtessel designed the sliding mode control system of $\mathrm{X}-33$ based on the time-scale separation principle, which has achieved the great control performance [7-9]. On the basis, the designer estimated the real-time perturbation and interference through introducing the sliding mode observer, which not only could suppress the buffeting, but also could improve the robust performance of system. In case of solving the buffeting problem of sliding mode control method effectively, it could be one of the efficient methods to solve the uncertain nonlinear system.

Linear parameter varying (LPV) control methodology [10] is an extension of $H_{\infty}$ control theory [11, 12] to the linear varying parameter system. To the nonlinear flight control system, many scholars have studied the application of LPV control methodology [13-15]. Nevertheless, using LPV control methodology needs to transform the nonlinear system to the LPV system, there is no uniform evaluation criterion to judging the approximative transform.

Nonlinear dynamic inversion (NDI) is the general nonlinear control strategy and methodology. Snell et al. [16] applied NDI to the supermaneuverable aircraft firstly. Bugajski and Enns [17] designed the universal architecture of flight control system design, the core of which was the structure block. Using the above method designed the control law of the large angle of attack aircraft. The simulation results verified that this methodology could satisfy the performance requirement of supermaneuvering flight control, but it must 

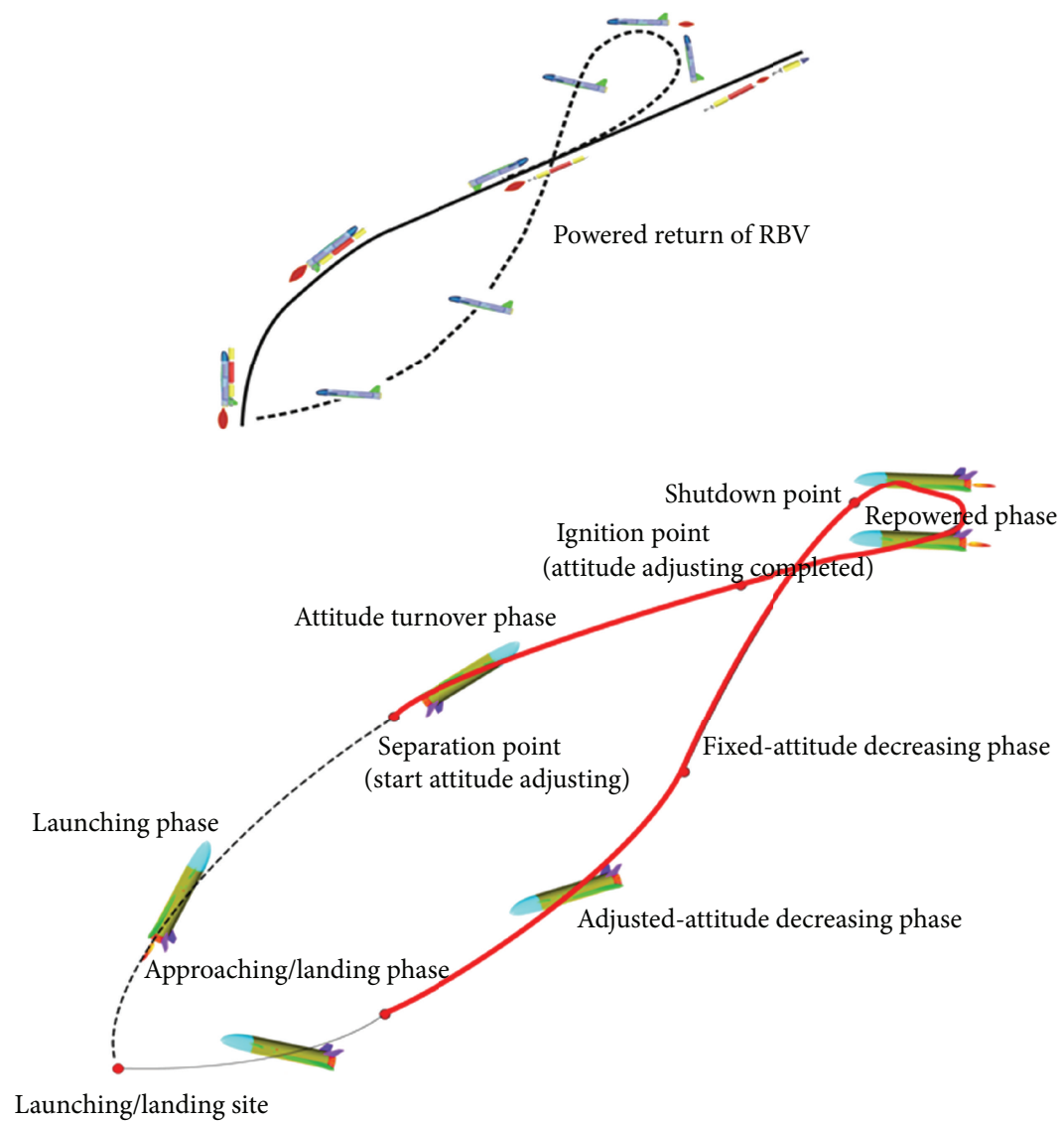

FIgURE 1: Flight profile of RBV.

be pointed out that modeling error and serious unusual aerodynamic effect cause the decreased robustness in the NDI control law. Farland and D'Souza [18] designed the attitude control system of lifting body reentry vehicle by NDI. The writer indicated that, to the parameter perturbation and external interference, using robust control theory strengthens the robustness of system under the NDI. NDI control methodology requires the accurate model. In fact it is impossible. Hence, it is necessary to combine the other control methods to eliminate the influence of inaccurate model.

\section{Flight Timing and Trajectory of RBV}

The RBV in this paper is the reusable vehicle which uses the rocket back to the launch site. Its flight processes as follows: after the separation from the disposable core stage (upper stage), RBV adjusts the attitude under RCS system in the tail during the sliding by inertia itself. When attitude angle of RBV reaches about $180^{\circ}$, the adjust phase finishes, and the head of RBV points to the direction of returning to the launch site. After the adjusting phase, with secondary ignition of RBV rocket engine (restricting the overload of secondary shut-off point), varying thrust regulator is used to adjust the flight velocity to satisfy the constraint condition of reentry. Afterwards, the vehicle experiences the fixed attitude dropping phase and the adjusting attitude phase and then enters the energy management and autonomous landing phase. The flight timing and trajectory of the RBV are shown in Figure 1.

Reusable boosted vehicle (RBV) experiences the subsonic, transonic, and supersonic phase during the whole flight, which the aerodynamic coefficients, dynamic pressure, and flight altitude change acutely. For this, the differences of the dynamic characteristics of RBV are significant during all of the flight phase. Besides that, there is a great different between the aerodynamic characteristics of RBV and the conventional vehicle. This paper focuses on the large attitude adjusting phase after the RBV separating between the core stages. To implement the high reliability and precision of $\mathrm{RBV}$, the nonlinear model is built according to the flight environment.

\section{RBV System Modeling}

This paper examines the problem of a nonaxisymmetric airframe which is flown in a large angle of attack. In the trajectory tracking problem (see Figure 2, body coordinate system of RBV), and the guidance law produces acceleration commands in the body $y$ and $z$ axes based on nominal trajectory. These acceleration commands can be converted into commands in roll angle and angle of attack, which are 


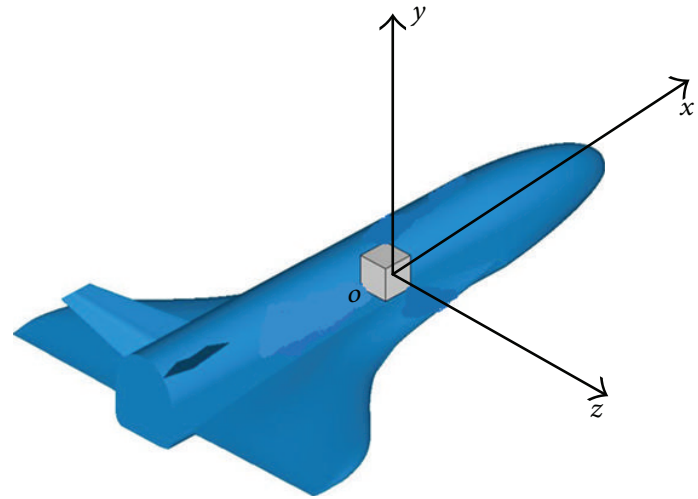

FIGURE 2: Body coordinate system of RBV.

fed into the autopilot. The task of the controller is to track commands in angle of attack and roll angle, while keeping sideslip angle small. There are many examples of angle of attack autopilots in the literature. The reader is referred to $[16,19]$ for treatments of autopilots that control angle of attack.

3.1. Dynamic Model for Attitude Adjusting Phase of $R B V$. Considering the assumption of designing the flight control system of X-33 [20], HOPE-X Series [21-23], the rigid-body nonlinear equations of motion for RBV of constant mass based on the body coordinate system of RBV and According to the coordinate system definition of [24], the rigid-body nonlinear equations of motion for RBV of constant mass is as follows:

$$
\begin{gathered}
\dot{\boldsymbol{\Omega}}=\mathbf{f}_{s}(\boldsymbol{\Theta}, \boldsymbol{\Omega})+\mathbf{g}_{s 2}(\boldsymbol{\Theta}, \boldsymbol{\Omega}) \mathbf{w}+\mathbf{g}_{s 1}(\boldsymbol{\Theta}, \boldsymbol{\Omega}) \boldsymbol{\delta}, \\
\dot{\mathbf{w}}=\mathbf{f}_{f}(\boldsymbol{\Theta}, \boldsymbol{\Omega}, \mathbf{w})+\mathbf{g}_{f}(\boldsymbol{\Theta}, \boldsymbol{\Omega}, \mathbf{w}) \mathbf{M}_{c},
\end{gathered}
$$

where $\boldsymbol{\Omega}=\left[\begin{array}{lll}\alpha & \beta & \gamma\end{array}\right]^{T}$ and $\mathbf{w}=\left[\begin{array}{lll}w_{x} & w_{y} & w_{z}\end{array}\right]^{T}$ are the system state variable and then $\Omega=\left[\begin{array}{lll}\alpha & \beta & \gamma\end{array}\right]^{T}$ are also the system output; $\boldsymbol{\delta}=\left[\begin{array}{ll}\delta_{\text {aero }} & \delta_{\text {rcs }}\end{array}\right]^{T}$ and $\mathbf{M}_{c}=\left[\begin{array}{lll}M_{c x} & M_{c y} & M_{c z}\end{array}\right]^{T}$ are the control variable; $\boldsymbol{\Theta}=\left[\begin{array}{llll}V & \gamma_{v} & \psi & \theta\end{array}\right]$ is the parameter vector; $\mathbf{f}_{s}(\boldsymbol{\Theta}, \boldsymbol{\Omega})=\left[\begin{array}{lll}f_{\alpha} & f_{\beta} & f_{\gamma}\end{array}\right]^{T}$ and $\mathbf{f}_{f}(\boldsymbol{\Theta}, \boldsymbol{\Omega}, \mathbf{w})=$ $\left[\begin{array}{lll}f_{w_{x}} & f_{w_{y}} & f_{w_{z}}\end{array}\right]^{T}$ are the nonlinear differential function; $\mathbf{g}_{s 2}(\boldsymbol{\Theta}, \boldsymbol{\Omega}) \in \mathbf{R}^{3 \times 3}$ and $\mathbf{g}_{f}(\boldsymbol{\Theta}, \boldsymbol{\Omega}, \mathbf{w}) \in \mathbf{R}^{3 \times 3}$ are the control matrix; $\mathbf{g}_{s 1}(\Theta, \Omega)$ represents the deflections of aerodynamic surfaces and control moment of RCS.

3.2. Aerodynamic Data Model of $R B V$. In order to design the flight control system of RBV in this paper conveniently, aerodynamic data models of RBV are built in the velocity coordinate system, and body coordinate system respectively [25].
Aerodynamic coefficient in the velocity coordinate system is as follows:

$$
\begin{aligned}
C_{x}= & C_{x 0}(\alpha, \beta, \mathrm{Ma})+C_{x \alpha}(\alpha, \mathrm{Ma}) \alpha \\
& +C_{x \delta_{\text {aero }}}\left(\alpha, \beta, \mathrm{Ma}, \delta_{\text {aero }}\right) \delta_{\text {aero }}+\Delta C_{x}(\alpha, \mathrm{Ma}), \\
C_{y}= & C_{y 0}(\alpha, \beta, \mathrm{Ma})+C_{y \alpha}(\alpha, \mathrm{Ma}) \alpha \\
& +C_{y \delta_{\text {aero }}}\left(\alpha, \beta, \mathrm{Ma}, \delta_{\text {aero }}\right) \delta_{\text {aero }}+\Delta C_{y}(\alpha, \mathrm{Ma}), \\
C_{z}= & C_{z 0}(\alpha, \beta, \mathrm{Ma})+C_{z \beta}(\alpha, \beta, \mathrm{Ma}) \beta \\
& +C_{z \delta_{\text {aero }}}\left(\alpha, \beta, \mathrm{Ma}, \delta_{\text {aero }}\right) \delta_{\text {aero }}+\Delta C_{z}(\alpha, \mathrm{Ma}),
\end{aligned}
$$

where $\Delta(\cdot)$ is the term of aerodynamic perturbation; Ma is the Mach number; $\delta_{\text {aero }}$ is the command of aerodynamic surfaces.

Aerodynamic moment coefficient in the body coordinate system:

$$
\begin{aligned}
m_{x}= & m_{x 0}(\alpha, \beta, \mathrm{Ma}) \\
& +\left(\frac{b}{2 V} m_{x w_{x}}\left(\alpha, \mathrm{Ma}_{a}\right) w_{x}+\frac{b}{2 V} m_{x w_{y}}(\alpha, \mathrm{Ma}) w_{y}\right) \\
& +m_{x \beta}(\beta, \mathrm{Ma}) \beta+m_{x \delta_{\text {aero }}}\left(\alpha, \beta, \mathrm{Ma}, \delta_{\text {aero }}\right) \\
& +\Delta m_{x}(\alpha, \mathrm{Ma}), \\
m_{y}= & m_{y 0}(\alpha, \beta, \mathrm{Ma})+m_{y \delta_{\text {aero }}}\left(\alpha, \beta, \mathrm{Ma}, \delta_{\text {aero }}\right) \delta_{\text {aero }} \\
& +\frac{b}{2 V}\left(m_{y w_{y}}(\alpha, \mathrm{Ma}) w_{y}+\frac{b}{2 V} m_{y w_{x}}(\alpha, \mathrm{Ma}) w_{x}\right) \\
& +m_{y \beta}(\beta, \mathrm{Ma}) \beta+\Delta m_{y}(\alpha, \mathrm{Ma}), \\
m_{z}= & m_{z 0}(\alpha, \beta, \mathrm{Ma})+\frac{c}{2 V} m_{z w_{z}}(\alpha, \mathrm{Ma}) w_{z} \\
& +m_{z \delta_{\text {aero }}}\left(\alpha, \beta, \mathrm{Ma}, \delta_{\text {aero }}\right) \delta_{\text {aero }} \\
& +m_{z \alpha}(\alpha, \mathrm{Ma}) \alpha+\Delta m_{z}(\alpha, \mathrm{Ma}),
\end{aligned}
$$

where $\Delta(\cdot)$ is the term of aerodynamic perturbation; $b$ is the span; $c$ is the mean aerodynamic chord.

\section{Robust Adaptive Inversion Control Based on Neural Network}

4.1. Single Hidden Layer Neural Network. The structure of the single hidden layer $[26,27]$ is shown in Figure 3, the input and output of which are defined:

$$
\begin{gathered}
\overline{\overline{\mathbf{x}}}=\left[\begin{array}{ll}
b_{V} & \overline{\mathbf{x}}^{T}
\end{array}\right]^{T}, \\
\mathbf{v}_{\mathrm{ad}}(\mathbf{W}, \mathbf{V}, \overline{\overline{\mathbf{x}}})=\mathbf{W}^{T} \boldsymbol{\sigma}\left(\mathbf{V}^{T} \overline{\overline{\mathbf{x}}}\right),
\end{gathered}
$$




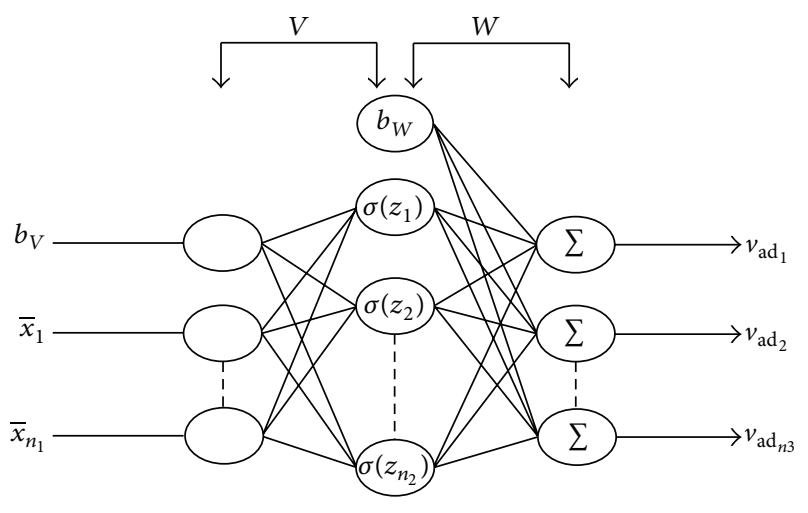

FIGURE 3: The structure of the single hidden neural network.

where $\overline{\mathbf{x}} \in \mathbf{R}^{n_{1} \times 1}$ is the input of neural network, which belongs to the certain compact set $\mathbf{D}_{\overline{\mathbf{x}}}$; the output of neural network is $\mathbf{v}_{\mathrm{ad}} \in \mathbf{R}^{n_{3} \times 1} ; \mathbf{V} \in \mathbf{R}^{\left(n_{1}+1\right) \times n_{2}}$ and $\mathbf{W} \in \mathbf{R}^{\left(n_{2}+1\right) \times n_{3}}$ are the weight of the input layer to hidden layer and the hidden layer to the output layer, respectively; $n_{1}, n_{2}$, and $n_{3}$ are the number of the input, the neurons in the hidden layer, and the output respectively.

Action function $\sigma(\cdot)$ is chosen Sigmoid function and

$$
\boldsymbol{\sigma}(z)=\left[\begin{array}{lllll}
b_{W} & \sigma\left(z_{1}\right) & \sigma\left(z_{2}\right) & \cdots & \sigma\left(z_{n_{2}}\right)
\end{array}\right]^{T},
$$

where

$$
\mathbf{z}=\mathbf{V}^{T} \overline{\overline{\mathbf{x}}}
$$

In addition, the offset term $b_{V}, b_{W} \geq 0$ in (4) and (6), the objective of which is to contain the threshold value of neurons in the hidden layer and to contain the output layer to the weight matrix, to implement the real-time adjusting of the threshold value of neurons.

4.2. Nonlinear Dynamic Inversion. The basic idea of the nonlinear dynamic inversion method [28-30] is to transform the dynamics of nonlinear system to a linear one through the inverse system of the controlled object and then implement the integrated system under the theory of the linear system. The general nonlinear system is described as follows:

$$
\begin{gathered}
\dot{\mathbf{x}}=\mathbf{f}(\mathbf{x}, \mathbf{u}), \\
\mathbf{y}=\mathbf{h}(\mathbf{x}),
\end{gathered}
$$

where $\mathbf{x} \in \mathbf{R}^{n}$ is the system state variable; $\mathbf{u} \in \mathbf{R}^{m}$ is the system control variable; $\mathbf{y} \in \mathbf{R}^{l}$ is the system output variable.

The objective of control system design of nonlinear system as (8) is to seek the control input $\mathbf{u}$, through which the system output $\mathbf{y}$ could track the expected output and timevarying trajectory $\mathbf{y}_{d}$ according to the certain precision and ensure the all the state variable of the closed loop be bounded.

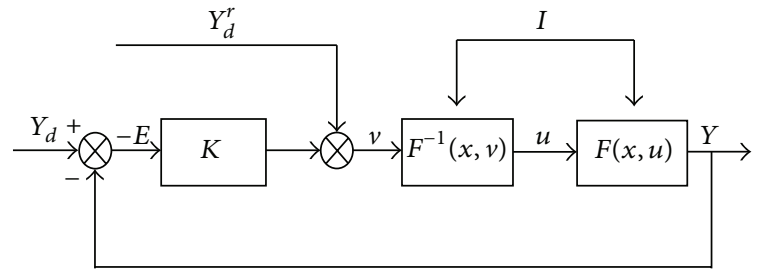

FIGURE 4: The diagram of dynamic inversion control method.

Performing the derivation to (8),

$$
\begin{gathered}
\mathbf{Y}=\left[\begin{array}{c}
y_{1}^{\left(r_{1}\right)} \\
y_{2}^{\left(r_{2}\right)} \\
\vdots \\
y_{p}^{\left(r_{p}\right)}
\end{array}\right]=\mathbf{F}(\mathbf{x}, \mathbf{u}), \\
\mathbf{u}=\mathbf{F}^{-1}(\mathbf{x}, \mathbf{v}) .
\end{gathered}
$$

Under the above control, the result of the $i$ th output subsystem is as follows:

$$
y_{i}^{\left(r_{i}\right)}=v_{i}
$$

where $v_{i}$ is called pseudocontrol input variable generally. The conventional nonlinear system in (8) could be transformed the linear system in (11) through (9) (10).

Defining the tracking error $e_{i}=y_{i}-y_{d i}$ and choosing the pseudocontrol variable:

$$
v_{i}=y_{d i}^{\left(r_{i}\right)}-k_{i r_{i}} e_{i}^{\left(r_{i}-1\right)}-\cdots-k_{i r} e_{i} .
$$

Hence, the dynamic equation of tracking error in the $i$ th closed loop subsystem is as follows:

$$
e_{i}^{\left(r_{i}\right)}+k_{i r} e_{i}^{\left(r_{i}-1\right)}+\cdots+k_{i r} e_{i}=0
$$

Selecting the coefficients $k_{i 1}, k_{i 2}, \ldots, k_{i r_{i}}>0$ to configurate all characteristic roots of $s^{r_{1}}+k_{i r_{i}} s^{r_{1}-1}+\cdots+k_{i 1}=0$ lying on the open left plane, namely, to ensure the stability of the $i$ th closed loop subsystem. The dynamic equation of tracking error in the entire closed loop system is as follows:

$$
\dot{\mathbf{E}}=-\mathbf{K E} \text {, }
$$

where the definitions of $\mathbf{K}$ and $\mathbf{E}$ are referenced in [31]. The block diagram of dynamic inverse method is shown in Figure 4 .

4.3. System Control Strategy. In (8), there are many inexact factors in the nonlinear system such as unmodeled dynamic, parameter perturbation, and external disturbance. Considering that the nonlinear dynamic inversion needs the accurate system model, the control strategy to counteract the model uncertainty must be proposed to guarantee the system tracking performance based on the nonlinear dynamic inversion method. 
Let the nominal value of $\mathbf{F}(\mathbf{x}, \mathbf{u})$ be $\widehat{\mathbf{F}}(\mathbf{x}, \mathbf{u})$; (10) is:

$$
\begin{aligned}
\mathbf{F}(\mathbf{x}, \mathbf{u}) & =\widehat{\mathbf{F}}(\mathbf{x}, \mathbf{u})+\Delta(\mathbf{x}, \mathbf{u}), \\
\mathbf{u} & =\widehat{\mathbf{F}}^{-1}(\mathbf{x}, \mathbf{v}) .
\end{aligned}
$$

Transforming the system uncertainty to the system inverse error $\Delta(\mathbf{x}, \mathbf{v})$,

$$
\Delta(\mathbf{x}, \mathbf{v})=\mathbf{F}\left(\mathbf{x}, \widehat{\mathbf{F}}^{-1}(\mathbf{x}, \mathbf{v})\right)-\widehat{\mathbf{F}}\left(\mathbf{x}, \widehat{\mathbf{F}}^{-1}(\mathbf{x}, \mathbf{v})\right) .
$$

The dynamic equation of the tracking error in entire closed loop system is

$$
\dot{\mathrm{E}}=\mathbf{A E}+\mathbf{B} \Delta(\mathbf{x}, \mathbf{v}),
$$

where $\mathbf{A}=-\mathbf{K}$.

Use the nonlinear approximation of neural network maps the inverse error $\Delta(\mathbf{x}, \mathbf{v})$ resulting from the system uncertainty to compensate the uncertain model [31]. Meanwhile, the error caused by the approximation inverse error of the neural network is suppressed by robust adaptive method. Hence, the definition of the robust adaptive inverse control strategy based on neural network is as follows:

$$
\begin{gathered}
\mathbf{u}=\widehat{\mathbf{F}}^{-1}(\mathbf{x}, \mathbf{v}), \\
\mathbf{v}=\mathbf{Y}_{d}^{r}+\mathbf{v}_{\mathrm{sc}}-\mathbf{v}_{\mathrm{ad}}+\mathbf{v}_{\mathrm{rb}},
\end{gathered}
$$

where

$$
\mathbf{v}_{\mathrm{sc}}=\mathrm{AE},
$$

where $\mathbf{u}$ is the actual system control input; $\mathbf{v}$ is the virtual system control input; $\mathbf{Y}_{d}^{r}$ is the $r$ order derivative of system reference input, which is similar to definition in [32]; $\mathbf{v}_{\mathrm{sc}}$ is the static control compensator; $\mathbf{v}_{\mathrm{ad}}$ is the neural network output; $\mathbf{v}_{\mathrm{rb}}$ is the robust adaptive term.

Under effecting of the control strategy (18), the dynamic error equation of closed loop from (19) is

$$
\dot{\mathbf{E}}=\mathbf{A E}+\mathbf{B}\left[\mathbf{v}_{\mathrm{ad}}-\mathbf{v}_{\mathrm{rb}}-\Delta(\mathbf{x}, \mathbf{v})\right] .
$$

The robust adaptive inversion control based on neural network is shown in Figure 5.

Based on the above theory, the control law design of the entire closed loop system is made up by the following three steps:

(1) Firstly, the static compensator $\mathbf{v}_{\mathrm{sc}}$ designed by nonlinear dynamic inversion method is exponent stable in the nominal neighborhood and satisfies the performance requirements of closed loop system in the nominal condition (i.e., $\Delta=0$ );

(2) Secondly, according to the strong nonlinear mapping ability of neural network, the inverse error $\Delta(\mathbf{x}, \mathbf{v})$ mapped by uncertainty $\Delta(\mathbf{x}, \mathbf{u})$ is approximated by neural network output $\mathbf{v}_{\text {ad }}$ when uncertainty condition exists.

(3) Finally, designing the robust adaptive control term $\mathbf{v}_{\mathrm{rb}}$ overcomes the influence of approximation error, then a better adaptive robust performance of entire closed loop system is achieved.

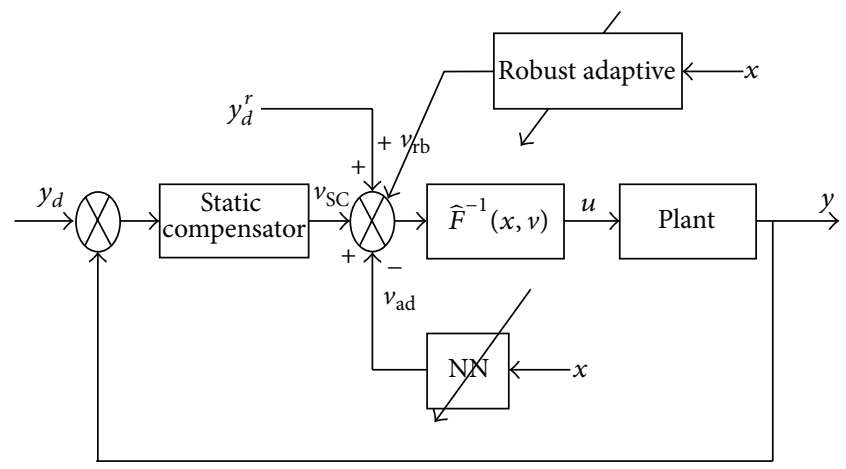

FIGURE 5: Robust adaptive inversion control based on neural network.

\section{Analysis and Design of Control System}

Based on the control strategy in the above third chapter, the neural network robust adaptive inversion control law is designed. And then, the stability of the closed loop system is proved strictly in the theory. Here, the input of SHLNN is $\overline{\mathbf{x}}=\left[\begin{array}{lll}\mathbf{E}^{T} & \mathbf{x}_{d} & \dot{\mathbf{x}}_{d}\end{array}\right]^{T}$, where $\mathbf{E}=\mathbf{x}-\mathbf{x}_{d}, \mathbf{x}$ is the system state, $\mathbf{x}_{d}$ is the expected system state, and $\mathbf{v}_{\text {ad }}$ is the neural network output.

Assumption 1. Giving arbitrary $\psi_{\varepsilon}^{*}>0$, there are the ideal neural network weight matrixes $\mathbf{V}^{*}$ and $\mathbf{W}^{*}$ to make the single hidden neural network uniform approximating the inverse error function $\Delta(\mathbf{x}, \mathbf{v})$ which is continuous and derivable in the compact set $\mathbf{D}_{\overline{\overline{\mathbf{x}}}}$. That is,

$$
\mathbf{W}^{* T} \boldsymbol{\sigma}\left(\mathbf{V}^{* T} \overline{\overline{\mathbf{x}}}\right)=\boldsymbol{\Delta}(\mathbf{x}, \mathbf{v})-\boldsymbol{\varepsilon}(\overline{\overline{\mathbf{x}}}),
$$

where $(\mathbf{x}, \mathbf{v}) \in \mathbf{D}_{\overline{\overline{\mathbf{x}}}} \subset \mathbf{R}^{n} \times \mathbf{R} ; \overline{\overline{\mathbf{x}}}=\left[\begin{array}{ll}1 & \overline{\mathbf{x}}^{T}\end{array}\right]^{T} ; \boldsymbol{\varepsilon}(\overline{\overline{\mathbf{x}}})$ is the approximation error and satisfies

$$
|\boldsymbol{\varepsilon}(\overline{\overline{\mathbf{x}}})| \leq \psi_{\varepsilon}^{*} .
$$

Assumption 2. Generally speaking, the ideal neural network weight matrixes $\mathbf{V}^{*}$ and $\mathbf{W}^{*}$ are unknown and may be not only. Therefore $\mathbf{V}^{*}$ and $\mathbf{W}^{*}$ can be defined:

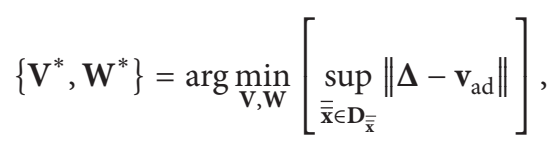

where

$$
\left\|\mathbf{V}^{*}\right\|_{F} \leq \overline{\overline{\mathbf{V}}} \quad\left\|\mathbf{W}^{*}\right\|_{F} \leq \overline{\overline{\mathbf{W}}}
$$

where $\|\cdot\|_{F}$ is Frobenius norm and $\overline{\overline{\mathbf{V}}}, \overline{\overline{\mathbf{W}}}$ are the positive constant.

For ideal weight matrixes $\mathbf{V}^{*}$ and $\mathbf{W}^{*}$ being unknown, let $\widehat{\mathbf{V}}$ and $\widehat{\mathbf{W}}$ be defined to describe the estimated value of neural network ideal weight matrix. $\mathbf{V}_{0}$ and $\mathbf{W}_{0}$ are the initial value of network, where $\widetilde{\mathbf{V}}=\widehat{\mathbf{V}}-\mathbf{V}^{*}$ and $\widetilde{\mathbf{W}}=\widehat{\mathbf{W}}-\mathbf{W}^{*}$ are the estimated error of neural network weight, respectively. 
Based on this estimated value, the online neural network approximation $\mathbf{v}_{\mathrm{ad}}$ is

$$
\mathbf{v}_{\mathrm{ad}}=\widehat{\mathbf{W}}^{T} \boldsymbol{\sigma}\left(\widehat{\mathbf{V}}^{T} \overline{\overline{\mathbf{x}}}\right)
$$

Now the dynamic error equation of closed loop system is

$$
\dot{\mathbf{E}}=\mathbf{A E}+\mathbf{B}\left[\widehat{\mathbf{W}}^{T} \boldsymbol{\sigma}\left(\widehat{\mathbf{V}}^{T} \overline{\overline{\mathbf{x}}}\right)-\mathbf{W}^{* T} \boldsymbol{\sigma}\left(\mathbf{V}^{* T} \overline{\overline{\mathbf{x}}}\right)-\mathbf{v}_{\mathrm{rb}}-\boldsymbol{\varepsilon}(\overline{\overline{\mathbf{x}}})\right]
$$

As $\mathbf{A}$ is Hurwitz matrix, there exists only positive definite matrix $\mathbf{P}$ with respect to the following Lyapunov function, which satisfies

$$
\mathbf{A}^{T} \mathbf{P}+\mathbf{P A}=-\mathbf{Q}
$$

where $\mathbf{Q}$ is an arbitrary positive definite matrix.

Considering the Taylor expansion of $\boldsymbol{\sigma}\left(\mathbf{V}^{* T} \overline{\overline{\mathbf{x}}}\right)$ in $\widehat{\mathbf{V}}^{T} \overline{\overline{\mathbf{x}}}$,

$$
\boldsymbol{\sigma}\left(\mathbf{V}^{*} \overline{\overline{\mathbf{x}}}\right)=\boldsymbol{\sigma}\left(\widehat{\mathbf{V}}^{T} \overline{\overline{\mathbf{x}}}\right)-\boldsymbol{\sigma}^{\prime}\left(\widehat{\mathbf{V}}^{T} \overline{\overline{\mathbf{x}}}\right) \overline{\mathbf{V}}^{T} \overline{\overline{\mathbf{x}}}+\mathcal{O}\left(\widetilde{\mathbf{V}}^{T} \overline{\overline{\mathbf{x}}}\right)^{2}
$$

Here, let $\mathbf{z}=\mathbf{V}^{* T} \overline{\overline{\mathbf{x}}}, \widehat{\mathbf{z}}=\widehat{\mathbf{V}}^{T} \overline{\overline{\mathbf{x}}}$

$$
\boldsymbol{\sigma}^{\prime}(\widehat{\mathbf{z}})=\left.\frac{d \boldsymbol{\sigma}(\mathbf{z})}{d \mathbf{z}}\right|_{\mathbf{z}=\widehat{\mathbf{z}}}=\left[\begin{array}{ccc}
0 & \cdots & 0 \\
\frac{\partial \sigma\left(z_{1}\right)}{\partial z_{1}} & \cdots & \frac{\partial \sigma\left(z_{1}\right)}{\partial z_{n_{2}}} \\
\vdots & \ddots & \vdots \\
\frac{\partial \sigma\left(z_{n_{2}}\right)}{\partial z_{1}} & \cdots & \frac{\partial \sigma\left(z_{n_{2}}\right)}{\partial z_{n_{2}}}
\end{array}\right]_{\mathbf{z}=\widehat{\mathbf{z}}} .
$$

Hence, $\widehat{\mathbf{W}}^{T} \boldsymbol{\sigma}\left(\widehat{\mathbf{V}}^{T} \overline{\overline{\mathbf{x}}}\right)-\mathbf{W}^{* T} \boldsymbol{\sigma}\left(\mathbf{V}^{* T} \overline{\overline{\mathbf{x}}}\right)$ in (26) is changed to

$$
\begin{aligned}
& \widehat{\mathbf{W}}^{T} \boldsymbol{\sigma}\left(\widehat{\mathbf{V}}^{T} \overline{\overline{\mathbf{x}}}\right)-\mathbf{W}^{* T} \boldsymbol{\sigma}\left(\mathbf{V}^{* T} \overline{\overline{\mathbf{x}}}\right) \\
& =\widetilde{\mathbf{W}}^{T}\left(\widehat{\boldsymbol{\sigma}}-\widehat{\boldsymbol{\sigma}}^{\prime} \widehat{\mathbf{V}}^{T} \overline{\overline{\mathbf{x}}}\right)+\widehat{\mathbf{W}}^{T} \widehat{\boldsymbol{\sigma}}^{\prime} \widetilde{\mathbf{V}}^{T} \overline{\overline{\mathbf{x}}}+\mathbf{W},
\end{aligned}
$$

where

$$
\mathbf{w}=\widetilde{\mathbf{W}}^{T} \widehat{\boldsymbol{\sigma}}^{\prime} \mathbf{V}^{T} \overline{\overline{\mathbf{x}}}-\mathbf{W}^{* T} \mathcal{O}^{2}(\widetilde{\mathbf{V}})
$$

Synthesizing (30) and (31)

$$
\begin{aligned}
\mathbf{W}= & \mathbf{W}^{* T}(\widehat{\boldsymbol{\sigma}}-\boldsymbol{\sigma})-\mathbf{W}^{* T} \widehat{\boldsymbol{\sigma}}^{\prime} \widehat{\mathbf{V}}^{T} \overline{\overline{\mathbf{x}}}+\widehat{\mathbf{W}}^{T} \widehat{\boldsymbol{\sigma}}^{\prime} \mathbf{V}^{* T} \overline{\overline{\mathbf{x}}} \\
|\mathbf{w}| \leq & \left\|\mathbf{V}^{*}\right\|_{F} \| \overline{\overline{\mathbf{x}}} * \text { ones }\left(1, n_{3}\right) * \widehat{\mathbf{W}}^{T} \widehat{\boldsymbol{\sigma}}^{\prime} \|_{F} \\
& +\left\|\mathbf{W}^{*}\right\|\left\|\widehat{\boldsymbol{\sigma}}^{\prime} \widehat{\mathbf{V}}^{T} \overline{\overline{\mathbf{x}}}\right\|+\left\|\mathbf{W}^{*}\right\|_{1},
\end{aligned}
$$

where $\|\cdot\|_{F}$ is Frobenius norm; $\|\cdot\|$ is Euclid norm; $\|\cdot\|_{1}$ is 1 norm; ones $\left(1, n_{3}\right) \in \mathbf{R}^{1 \times n_{3}}$ is the 1 matrix. The upper bound of $w$ is expressed as the following form:

$$
|\mathbf{w}| \leq \psi_{w}^{*} s_{w}(\widehat{\mathbf{V}}, \widehat{\mathbf{W}}, \overline{\overline{\mathbf{x}}})
$$

where

$$
\begin{gathered}
\psi_{w}^{*}=\max \left\{\left\|\mathbf{V}^{*}\right\|_{F},\left\|\mathbf{W}^{*}\right\|,\left\|\mathbf{W}^{*}\right\|_{1}\right\}, \\
s_{w}(\widehat{\mathbf{V}}, \widehat{\mathbf{W}}, \overline{\overline{\mathbf{x}}})=\left\|\overline{\overline{\mathbf{x}}} * \operatorname{ones}\left(1, n_{3}\right) * \widehat{\mathbf{W}}^{T}\right\|_{F}+\left\|\widehat{\boldsymbol{\sigma}}^{\prime} \widehat{\mathbf{V}}^{T} \overline{\overline{\mathbf{x}}}\right\|+1 .
\end{gathered}
$$

Substituting (26) by Taylor expansion of network approximation inverse error, the dynamic error equation of the closed loop system is as follows:

$$
\begin{gathered}
\dot{\mathbf{E}}=\mathbf{A E}+\mathbf{B}\left[\widetilde{\mathbf{W}}^{T}\left(\widehat{\boldsymbol{\sigma}}-\widehat{\boldsymbol{\sigma}}^{\prime} \widehat{\mathbf{V}}^{T} \overline{\overline{\mathbf{x}}}\right)+\widehat{\mathbf{W}}^{T} \widehat{\boldsymbol{\sigma}}^{\prime} \widetilde{\mathbf{V}}^{T} \overline{\overline{\mathbf{x}}}\right. \\
\left.+\mathbf{w}+\mathbf{v}_{\mathrm{rb}}-\boldsymbol{\varepsilon}(\overline{\overline{\mathbf{x}}})\right] .
\end{gathered}
$$

Introducing the operator vec to the matrix $\mathbf{M} \in \mathbf{R}^{n \times m}$, in order to analysis the following theory conveniently,

$$
\operatorname{vec} \mathbf{M}=\left[\begin{array}{llll}
\operatorname{col}_{1}(\mathbf{M})^{T} & \operatorname{col}_{2}(\mathbf{M})^{T} & \cdots & \operatorname{col}_{m}(\mathbf{M})^{T}
\end{array}\right]^{T} \in \mathbf{R}^{n m},
$$

where $\operatorname{col}_{i}(\mathbf{M})$ is the $i$ th column of the matrix $\mathbf{M}$.

Let $\widetilde{\mathbf{Z}}=\left[\begin{array}{ccc}\widetilde{\mathbf{W}} & 0 & 0 \\ 0 & \widetilde{\mathbf{V}} & 0 \\ 0 & 0 & \widetilde{\psi}\end{array}\right], \widetilde{\psi}=\psi-\psi_{\max }^{*}$. Considering the composite errors vector $\boldsymbol{\varsigma}=\left[\begin{array}{c}\mathbf{E} \\ \operatorname{vec} \widetilde{\mathbf{Z}}\end{array}\right] \in \mathbf{D}_{\boldsymbol{\varsigma}}$, the compact set defined as follows is

$$
\mathbf{B}_{R}=\left\{\boldsymbol{\varsigma} \in \mathbf{D}_{\boldsymbol{\varsigma}}:\|\boldsymbol{\varsigma}\| \leq \mathbf{R}\right\}, \quad \mathbf{R}>0 .
$$

Assumption 3. $\mathbf{B}_{R}$ is the largest hypersphere in compact set $\mathrm{D}_{\boldsymbol{c}}$ and satisfies

$$
R>C \sqrt{\frac{\lambda_{\max }(\mathbf{T})}{\lambda_{\min }(\mathbf{T})}} \geq C>0,
$$

where $\lambda_{\max }(\mathbf{T})$ and $\lambda_{\min }(\mathbf{T})$ are the maximum and minimum characteristics value of the matrix $\mathbf{T}$, respectively. And the $\mathbf{T}$ is:

$$
\mathbf{T}=\frac{1}{2}\left[\begin{array}{cccc}
\mathbf{P} & 0 & 0 & 0 \\
0 & \boldsymbol{\Gamma}_{W}^{-1} & 0 & 0 \\
0 & 0 & \boldsymbol{\Gamma}_{V}^{-1} & 0 \\
0 & 0 & 0 & \gamma_{\psi}^{-1}
\end{array}\right]
$$

where $\boldsymbol{\Gamma}_{W}$ and $\boldsymbol{\Gamma}_{V}$ are positive definite matrixes and $\gamma_{\psi}>0$.

Let $v_{R}$ be the minimum value given by following function $V$ along the bound of hypersphere $\mathbf{B}_{R}$ :

$$
\begin{gathered}
V=\boldsymbol{\varsigma}^{T} \mathbf{T} \boldsymbol{\varsigma}, \\
v_{R}=\min _{\|\boldsymbol{c}\|=R} V(\boldsymbol{s}, t)=R^{2} \lambda_{\min }(\mathbf{T}) .
\end{gathered}
$$

Define the following compact set:

$$
\boldsymbol{\Omega}_{R}=\left\{\boldsymbol{\varsigma} \in \mathbf{B}_{R}: V \leq v_{R}\right\} .
$$

Theorem 4. To the nonlinear uncertain system composed by (8), (15), and (18), the following designed control law based on neural network robust adaptive dynamic inversion satisfies 
all the error signals $\boldsymbol{\varsigma}(t)$ uniformly bounded considering the conditions of Assumptions 1-3 when the initial value $\boldsymbol{\varsigma}(0)$ of composite errors belongs to the compact set $\Omega_{R}$. When $t>T_{0}$, the trajectory of arbitrary initial error signals from $\boldsymbol{\Omega}_{R}$ will enter the boundary of $\Omega_{C}$ in the finite time, the final value of

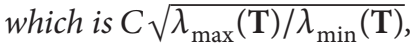

$$
\begin{gathered}
\mathbf{u}=\widehat{\mathbf{F}}^{-1}(\mathbf{x}, \mathbf{v}), \\
\mathbf{v}=\dot{\mathbf{X}}_{d}+\mathbf{v}_{s c}-\mathbf{v}_{a d}+\mathbf{v}_{r b},
\end{gathered}
$$

where $\boldsymbol{u}$ is the actual system control input; $\mathbf{v}$ is the virtual system control input; $\dot{\mathbf{X}}_{d}$ is the derivative of system reference input; $\mathbf{v}_{s c}$ is the static control compensator; $\mathbf{v}_{a d}$ is the neural network output; $\mathbf{v}_{r b}$ is the robust adaptive term, and

$$
\begin{gathered}
\mathbf{v}_{s c}=\mathbf{A E}, \\
\mathbf{v}_{a d}=\widehat{\mathbf{W}}^{T} \boldsymbol{\sigma}\left(\widehat{\mathbf{V}}^{T} \overline{\overline{\mathbf{x}}}\right), \\
\mathbf{v}_{r b}=\psi s^{*} \tanh \left(\frac{\xi^{T} s^{*}}{\Upsilon}\right),
\end{gathered}
$$

where $\mathbf{A}$ is Hurwitz matrix and $\boldsymbol{\xi}=\mathbf{E}^{\mathrm{T}} \mathbf{P B}$ and $\mathbf{B}=\mathbf{I}_{m}$ satisfy (26). The following neural network adaptive law and robust adaptive law are

$$
\begin{gathered}
\dot{\hat{\mathbf{V}}}=-\boldsymbol{\Gamma}_{V}\left[\overline{\overline{\mathbf{x}}} \boldsymbol{\xi} \widehat{\mathbf{W}}^{T} \widehat{\boldsymbol{\sigma}}^{\prime}+\lambda_{V}\left(\widehat{\mathbf{V}}-\mathbf{V}_{0}\right)\right], \\
\dot{\hat{\mathbf{W}}}=-\boldsymbol{\Gamma}_{W}\left[\left(\widehat{\boldsymbol{\sigma}}-\widehat{\boldsymbol{\sigma}}^{\prime} \widehat{\mathbf{V}}^{T} \overline{\overline{\mathbf{x}}}\right) \boldsymbol{\xi}+\lambda_{W}\left(\widehat{\mathbf{W}}-\mathbf{W}_{0}\right)\right], \\
\dot{\psi}=\gamma_{\psi}\left[\boldsymbol{\xi} s^{*} \tanh \left(\frac{\boldsymbol{\xi}^{T} s^{*}}{\Upsilon}\right)-\lambda_{\psi}\left(\psi-\psi_{0}\right)\right],
\end{gathered}
$$

where $s^{*}=1+s_{w} ; \psi_{\max }^{*}=\max \left\{\psi_{\varepsilon}^{*}, \psi_{w}^{*}\right\} ; \psi$ is the estimated value of $\psi_{\max }^{*} ; \psi_{0}$ is the initial value of $\psi ; \widetilde{\psi}=\psi-\psi_{\max }^{*} ; \Gamma_{V}$ and $\Gamma_{W}$ are positive definite matrixes; $\gamma_{\psi}>0, \lambda_{V}>0, \lambda_{W}>0$, and $\Upsilon>0$.

Proof. The Lyapunov function of system is

$$
\begin{aligned}
V= & \frac{1}{2} \mathbf{E}^{T} \mathbf{P E}+\frac{1}{2} \operatorname{tr}\left(\widetilde{\mathbf{W}}^{T} \boldsymbol{\Gamma}_{W}^{-1} \widetilde{\mathbf{W}}\right) \\
& +\frac{1}{2} \operatorname{tr}\left(\widetilde{\mathbf{V}}^{T} \boldsymbol{\Gamma}_{V}^{-1} \widetilde{\mathbf{V}}\right)+\frac{1}{2} \widetilde{\psi} \gamma_{\psi}^{-1} \widetilde{\psi}=\boldsymbol{\varsigma}^{T} \mathbf{T} \boldsymbol{\varsigma} .
\end{aligned}
$$

Solving the time derivative of (45) and using (27) and (35) yield

$$
\begin{aligned}
\dot{V}= & \xi\left[\widetilde{\mathbf{W}}^{T}\left(\widehat{\boldsymbol{\sigma}}-\widehat{\boldsymbol{\sigma}}^{\prime} \widehat{\mathbf{V}}^{T} \overline{\overline{\mathbf{x}}}\right)+\widetilde{\mathbf{W}}^{T} \widehat{\boldsymbol{\sigma}}^{\prime} \widetilde{\mathbf{V}}^{T} \overline{\overline{\mathbf{x}}}-\mathbf{v}_{\mathrm{rb}}+\mathbf{w}-\boldsymbol{\varepsilon}(\overline{\overline{\mathbf{x}}})\right] \\
& -\frac{1}{2} \mathbf{E}^{T} \mathbf{Q} \mathbf{E}+\operatorname{tr}\left(\widetilde{\mathbf{W}}^{T} \boldsymbol{\Gamma}_{W}^{-1} \dot{\overline{\mathbf{W}}}\right)+\operatorname{tr}\left(\widetilde{\mathbf{V}}^{T} \boldsymbol{\Gamma}_{V}^{-1} \dot{\tilde{\mathbf{V}}}\right)+\widetilde{\psi} \gamma_{\psi}^{-1} \dot{\tilde{\psi}}
\end{aligned}
$$

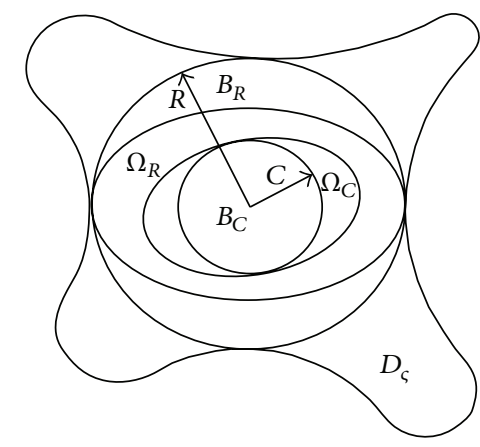

FIgURE 6: The geometric relation during sets.

Substituting the neural network robust adaptive dynamic inversion control law (42) (45) to (46) yields

$$
\begin{aligned}
\dot{V}= & -\frac{1}{2} \mathbf{E}^{T} \mathbf{Q E} \\
& +\boldsymbol{\xi}\left[\widetilde{\mathbf{W}}^{T}\left(\widehat{\boldsymbol{\sigma}}-\widehat{\boldsymbol{\sigma}}^{\prime} \widehat{\mathbf{V}}^{T} \overline{\overline{\mathbf{x}}}\right)+\widehat{\mathbf{W}}^{T} \widehat{\boldsymbol{\sigma}}^{\prime} \widetilde{\mathbf{V}}^{T} \overline{\overline{\mathbf{x}}}-\mathbf{v}_{\mathrm{rb}}+\mathbf{w}-\boldsymbol{\varepsilon}(\overline{\overline{\mathbf{x}}})\right] \\
& -\operatorname{tr}\left[\widetilde{\mathbf{W}}^{T}\left(\widehat{\boldsymbol{\sigma}}-\widehat{\boldsymbol{\sigma}}^{\prime} \widehat{\mathbf{V}}^{T} \overline{\overline{\mathbf{x}}}\right) \boldsymbol{\xi}+\lambda_{W} \widetilde{\mathbf{W}}^{T}\left(\widehat{\mathbf{W}}-\mathbf{W}_{0}\right)\right] \\
& -\operatorname{tr}\left[\widetilde{\mathbf{V}}^{T} \overline{\overline{\mathbf{x}}} \boldsymbol{\xi} \widehat{\mathbf{W}}^{T} \widehat{\boldsymbol{\sigma}}^{\prime}+\lambda_{V} \widetilde{\mathbf{V}}^{T}\left(\widehat{\mathbf{V}}-\mathbf{V}_{0}\right)\right] \\
& +\widetilde{\psi}\left[\xi s^{*} \tanh \left(\frac{\xi^{T} s^{*}}{\Upsilon}\right)-\lambda_{\psi}\left(\psi-\psi_{0}\right)\right] .
\end{aligned}
$$

Simplifying the above equation,

$$
\begin{aligned}
\dot{V}= & -\frac{1}{2} \mathbf{E}^{T} \mathbf{Q E} \\
& -\lambda_{W} \operatorname{tr}\left(\widetilde{\mathbf{W}}^{T}\left(\widehat{\mathbf{W}}-\mathbf{W}_{0}\right)\right)-\lambda_{V} \operatorname{tr}\left(\widetilde{\mathbf{V}}^{T}\left(\widehat{\mathbf{V}}-\mathbf{V}_{0}\right)\right) \\
& +\widetilde{\psi}\left[\boldsymbol{\xi}^{*} \tanh \left(\boldsymbol{\xi}^{T} s^{*}\right)-\lambda_{\psi}\left(\psi-\psi_{0}\right)\right] \\
& +\boldsymbol{\xi}\left[\mathbf{w}-\mathbf{v}_{\mathrm{rb}}-\boldsymbol{\varepsilon}(\overline{\mathbf{x}})\right]
\end{aligned}
$$

Amplifying (48) according to the conditions of $s^{*}=1+s_{w}$ and $\psi_{\max }^{*}=\max \left\{\psi_{\varepsilon}^{*}, \psi_{w}^{*}\right\}$ yields

$$
\begin{aligned}
\dot{V} \leq & -\frac{1}{2} \mathbf{E}^{T} \mathbf{Q E} \\
& -\lambda_{W} \operatorname{tr}\left(\widetilde{\mathbf{W}}^{T}\left(\widehat{\mathbf{W}}-\mathbf{W}_{0}\right)\right)-\lambda_{V} \operatorname{tr}\left(\widetilde{\mathbf{V}}^{T}\left(\widehat{\mathbf{V}}-\mathbf{V}_{0}\right)\right) \\
& +\|\boldsymbol{\xi}\| \psi_{\max }^{*} s^{*}-\xi \psi s^{*} \tanh \left(\frac{\boldsymbol{\xi}^{T} s^{*}}{\Upsilon}\right) \\
& +\widetilde{\psi}\left[\xi s^{*} \tanh \left(\frac{\xi^{T} s^{*}}{\Upsilon}\right)-\lambda_{\psi}\left(\psi-\psi_{0}\right)\right] .
\end{aligned}
$$




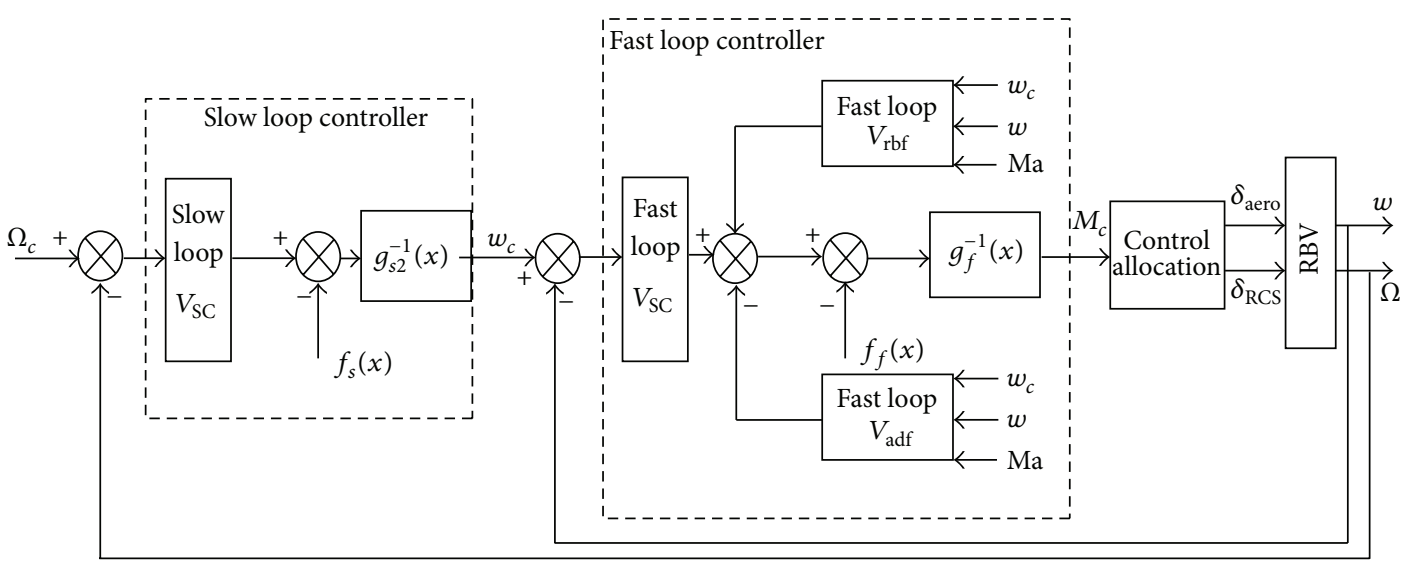

FIGURE 7: The structure diagram of RBV control system.

Simplifying (49) based on the given estimated error $\widetilde{\psi}=$ $\psi-\psi_{\max }^{*}$ yields

$$
\begin{aligned}
\dot{V} \leq & -\frac{1}{2} \mathbf{E}^{T} \mathbf{Q E} \\
& -\lambda_{W} \operatorname{tr}\left(\widetilde{\mathbf{W}}\left(\widehat{\mathbf{W}}-\mathbf{W}_{0}\right)\right)-\lambda_{V} \operatorname{tr}\left(\widetilde{\mathbf{V}}^{T}\left(\widehat{\mathbf{V}}-\mathbf{V}_{0}\right)\right) \\
& -\lambda_{\psi} \widetilde{\psi}\left(\psi-\psi_{0}\right)+\psi_{\max }^{*}\left[\|\xi\| s^{*}-\xi^{*} \tanh \left(\frac{\xi^{T} s^{*}}{\Upsilon}\right)\right] .
\end{aligned}
$$

Using the following inequality,

$$
0 \leq\|\zeta\|-\zeta \tanh \left(\frac{\zeta}{\Upsilon}\right) \leq \kappa_{1} \Upsilon
$$

Simplifying (50) yields

$$
\begin{aligned}
\dot{V} \leq & -\frac{1}{2} \lambda_{\min }(\mathbf{Q})\|\mathbf{E}\|^{2} \\
& -\lambda_{W} \operatorname{tr}\left(\widetilde{\mathbf{W}}^{T}\left(\widehat{\mathbf{W}}-\mathbf{W}_{0}\right)\right)+\psi_{\max }^{*} \kappa_{1} \Upsilon \\
& -\lambda_{V} \operatorname{tr}\left(\widetilde{\mathbf{V}}^{T}\left(\widehat{\mathbf{V}}-\mathbf{V}_{0}\right)\right)-\lambda_{\psi} \widetilde{\psi}\left(\psi-\psi_{0}\right),
\end{aligned}
$$

where $\lambda_{\min }(\mathbf{Q})$ is the minimum characteristics value of $\mathbf{Q}$.

Combining with $[33,34]$ :

$$
\begin{aligned}
\dot{V} \leq & -\frac{1}{2} \lambda_{\min }(\mathbf{Q})\|\mathbf{E}\|^{2} \\
& -\frac{\lambda_{W}}{2}\|\widetilde{\mathbf{W}}\|_{F}^{2}-\frac{\lambda_{V}}{2}\|\widetilde{\mathbf{V}}\|_{F}^{2}-\frac{\lambda_{\psi}}{2}|\widetilde{\psi}|^{2} \\
& +\frac{\lambda_{W}}{2}\left\|\mathbf{W}^{*}-\mathbf{W}_{0}\right\|_{F}^{2}+\frac{\lambda_{V}}{2}\left\|\mathbf{V}^{*}-\mathbf{V}_{0}\right\|_{F}^{2} \\
& +\frac{\lambda_{\psi}}{2}\left|\psi_{\text {max }}^{*}-\psi_{0}\right|^{2}+\psi_{\max _{1}}^{*} \kappa_{1} \Upsilon
\end{aligned}
$$

Giving definitions as follows:

$$
\begin{gathered}
\overline{\mathbf{Z}}=\frac{\lambda_{W}}{2}\left\|\mathbf{W}^{*}-\mathbf{W}_{0}\right\|_{F}^{2}+\frac{\lambda_{V}}{2}\left\|\mathbf{V}^{*}-\mathbf{V}_{0}\right\|_{F}^{2} \\
+\frac{\lambda_{\psi}}{2}\left|\psi_{\max }^{*}-\psi_{0}\right|^{2}+\psi_{\max }^{*} \kappa_{1} \Upsilon, \\
\widetilde{\mathbf{Z}}=\left[\begin{array}{ccc}
\widetilde{\mathbf{W}} & 0 & 0 \\
0 & \widetilde{\mathbf{V}} & 0 \\
0 & 0 & \widetilde{\psi}
\end{array}\right], \quad \lambda_{\min }=\frac{1}{2} \min \left(\lambda_{W}, \lambda_{V}, \lambda_{\psi}\right) .
\end{gathered}
$$

Equation (55) can be written:

$$
\dot{V} \leq-\frac{1}{2} \lambda_{\min }(\mathbf{Q})\|\mathbf{E}\|^{2}+\overline{\mathbf{Z}}-\lambda_{\min }\|\widetilde{\mathbf{Z}}\|_{F}^{2} .
$$

From (56), it is obvious that $\dot{V}<0$ when satisfying the following inequality:

$$
\begin{gathered}
\|\mathbf{E}\|>\sqrt{\frac{2 \overline{\mathbf{Z}}}{\lambda_{\text {min }}(\mathbf{Q})}}=C_{1}, \\
\|\widetilde{\mathbf{Z}}\|_{F}>\sqrt{\frac{\overline{\mathbf{Z}}}{\lambda_{\text {min }}}}=C_{2}, \\
\|\boldsymbol{s}\| \geq \max \left\{C_{1}, C_{2}\right\}=C .
\end{gathered}
$$

From (57), it is obvious that if we satisfy the $\dot{V}<0$, (58) must be established, which indicates that there is a compact set. In the out of the compact set, $\dot{V}<0$ is established. Defining the compact set proves that all of the error signals are ultimately bounded. Defining the following the hypersphere [35] firstly is

$$
\mathbf{B}_{C}=\left\{\boldsymbol{\varsigma} \in \mathbf{B}_{R}:\|\boldsymbol{\varsigma}\| \leq C\right\} .
$$

There is $\dot{V}<0$ beyond the compact set $\mathbf{B}_{C}$. From (44), it yields

$$
\mathbf{B}_{C} \subset \mathbf{B}_{R} .
$$



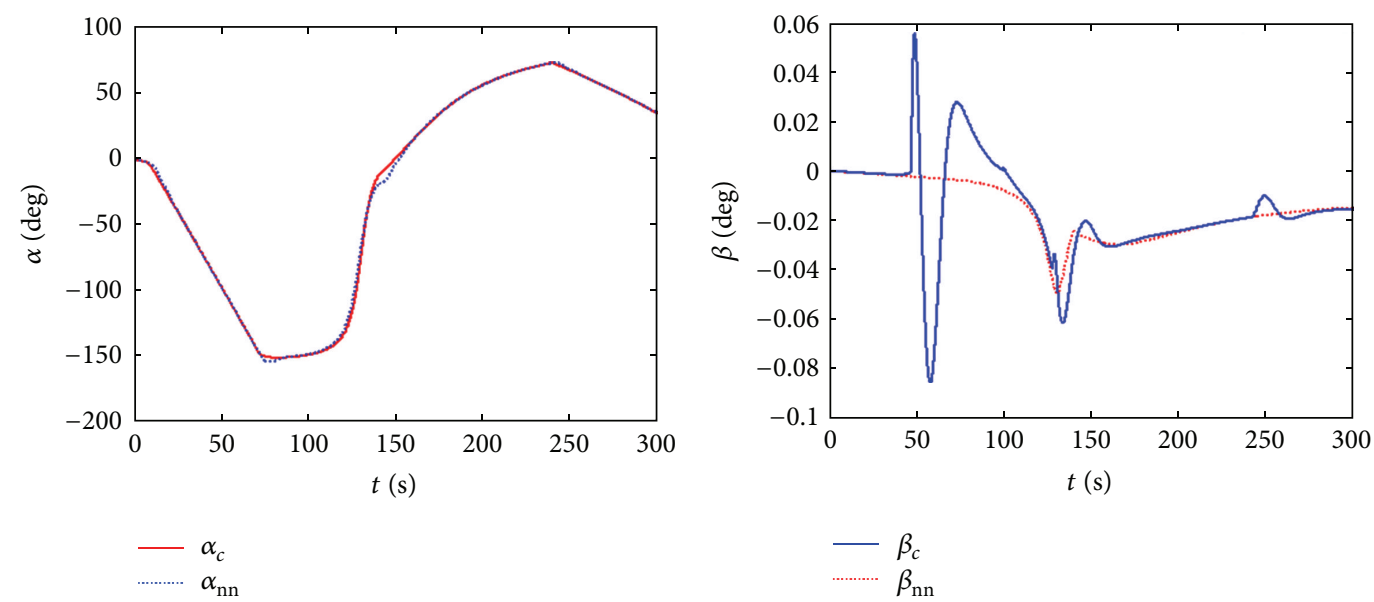

(a)
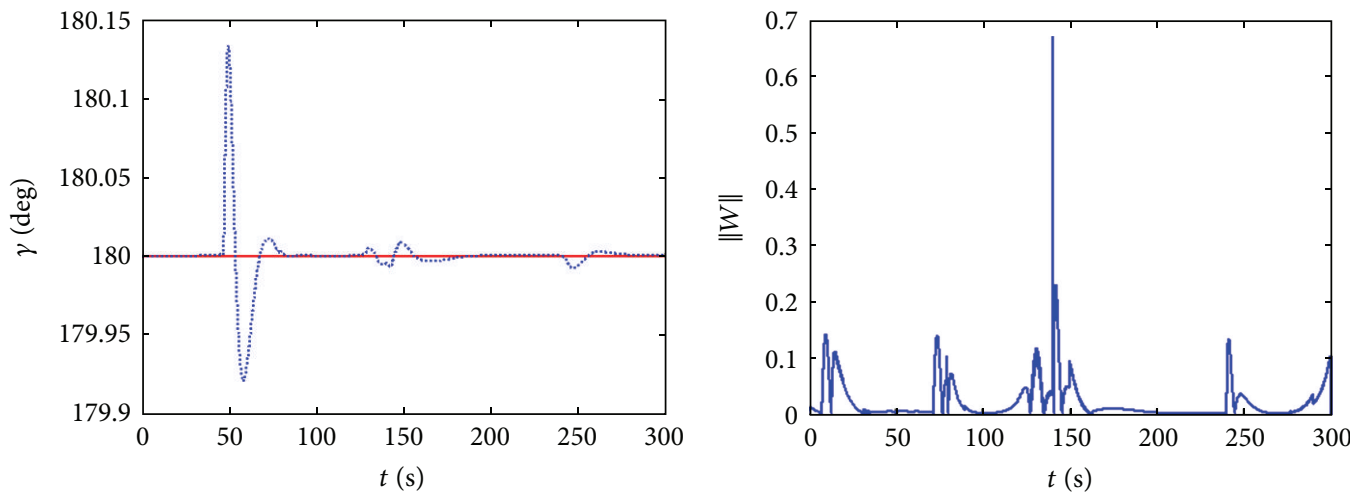

$-\gamma_{c}$

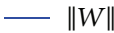

$\ldots \ldots \ldots \ldots \gamma_{\mathrm{nn}}$

(c)
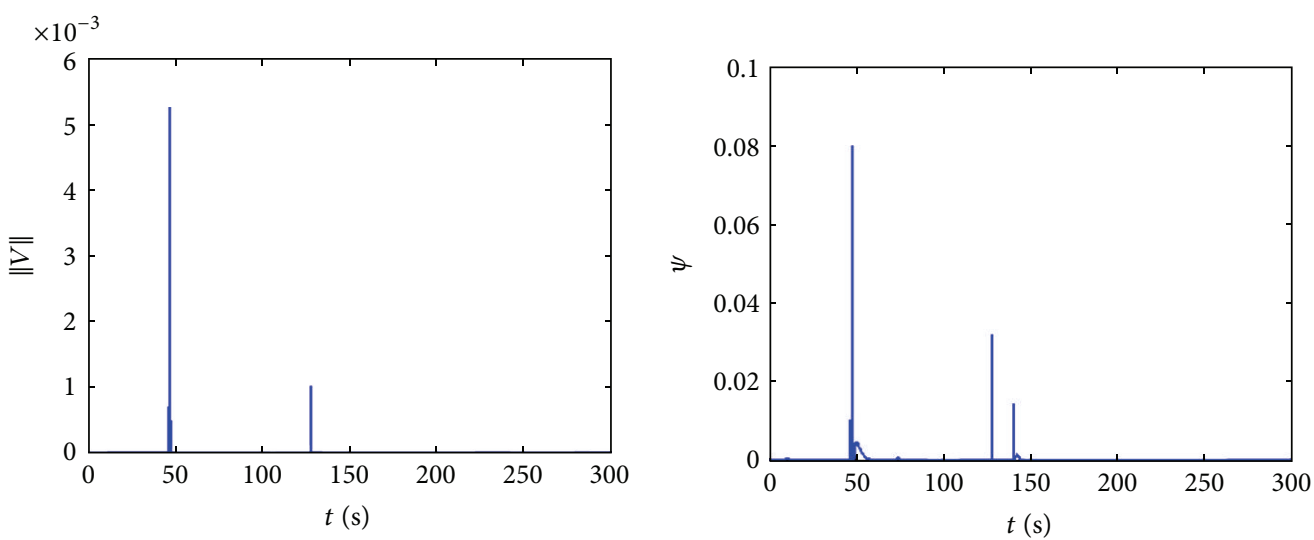

$-\|V\|$

(e)

(f)

FIGURE 8: The simulation curve of the whole flight based on the neural network robust adaptive inversion method.

Let $v_{C}$ be the maximum of function $V$ along the boundary of the hypersphere $\mathbf{B}_{C}$ :

$$
v_{C}=\min _{\|\boldsymbol{c}\|=C} V(\boldsymbol{s}, t)=C^{2} \lambda_{\max }(\mathbf{T}) .
$$

The compact set $\boldsymbol{\Omega}_{C}$ is

$$
\boldsymbol{\Omega}_{C}=\left\{\boldsymbol{\varsigma} \in \mathbf{B}_{C}: V \leq v_{C}\right\} .
$$

From (38) and Figure 6, it can be proved that $\mathbf{B}_{C} \subset \boldsymbol{\Omega}_{C} \subset$ $\boldsymbol{\Omega}_{R} \subset \mathbf{B}_{R}$. If the composite initial errors $\boldsymbol{\varsigma}(0) \in \boldsymbol{\Omega}_{R}$, all of the error signals are ultimately bounded in the closed loop system. According to the Lyapunov stability theorem, when $t>T_{0}$, the trajectory of arbitrary initial error signals from $\Omega_{R}$ will enter the boundary of $\Omega_{C}$ in the finite time, the final value of which is $C \sqrt{\lambda_{\max }(\mathbf{T}) / \lambda_{\min }(\mathbf{T})}$. The proof is finished. 


\section{Simulation and Validation of RBV}

On the basis of robust adaptive dynamic inversion control strategy in the above chapter and the established RBV model, the simulation verifies the proposed control law of neural network robust adaptive inversion validity. The perturbations are added to the simulation. The attitude tracking commands are produced by guidance module in simulation. When considering the uncertainty, the double loop system determined by (1) is described as follows:

$$
\begin{gathered}
\dot{\mathbf{x}}_{s}=\dot{\mathbf{\Omega}}=\mathbf{f}_{s}+\mathbf{g}_{s 2} \mathbf{w}+\Delta_{s}, \\
\mathbf{y}_{s}=\mathbf{\Omega}=\mathbf{x}_{s}, \\
\dot{\mathbf{x}}_{f}=\dot{\mathbf{w}}=\mathbf{f}_{f}+\mathbf{g}_{f} \mathbf{M}_{c}+\Delta_{f}, \\
\mathbf{y}_{f}=\mathbf{w}=\mathbf{x}_{f},
\end{gathered}
$$

where $\Delta_{f}=\left[\begin{array}{lll}\Delta_{w_{x}} & \Delta_{w_{y}} & \Delta_{w_{z}}\end{array}\right]^{T}, \Delta_{s}=\left[\begin{array}{lll}\Delta_{\alpha} & \Delta_{\beta} & \Delta_{\gamma}\end{array}\right]^{T}$ represent the uncertain factors, like the parameter perturbations and external interferences, in the fast loop, and slow loop respectively. To simplifying the design, assuming that $\Delta_{s}=0$, the neural network robust adaptive inversion control law to the fast loop is given. As the influence of fast loop is greater than the slow loop, the simplification is reasonable. According to the control mode of fast loop and slow loop of nonlinear dynamic inversion and the control law described in (42), the control input and output of fast loop when $\Delta_{f}$ exists are as follows:

$$
\begin{gathered}
\mathbf{w}_{c}=\mathbf{g}_{s_{2}}^{-1}\left(\dot{\boldsymbol{\Omega}}_{d}+\mathbf{w}_{s} \mathbf{e}_{\Omega}-\mathbf{f}_{s}\right), \\
\mathbf{M}_{c}=\mathbf{g}_{f}^{-1}\left(\dot{\mathbf{w}}_{d}+\overline{\mathbf{w}}_{k} \mathbf{e}_{w}-\mathbf{f}_{f}+\mathbf{v}_{\mathrm{rbf}}-\mathbf{v}_{\mathrm{adf}}\right),
\end{gathered}
$$

where $\dot{\mathbf{\Omega}}_{d}, \mathbf{e}_{\Omega}, \mathbf{w}_{s}, \mathbf{f}_{s}, \dot{\mathbf{w}}_{d}, \mathbf{e}_{w}, \overline{\mathbf{w}}_{k}$, and $\mathbf{f}_{f}$ reference [31]. $\mathbf{g}_{s_{2}}^{-1}$ and $\mathbf{g}_{f}^{-1}$ exist during flight process of RBV; $\mathbf{v}_{\text {rbf }}, \mathbf{v}_{\text {adf }}$ represent the robust adaptive term and approximation output of fast loop, respectively. The structure diagram of RBV control system is shown in Figure 7.

The parameter setting and initial condition of system simulation reference [31]. The simulation results of command angle tracking during large attitude adjusting phase are shown in Figure 8.

Figure 8 indicates the whole flight simulation curves of $\mathrm{RBV}$ control system during the turn period and reentry phase the $\left[\begin{array}{lll}\alpha_{c} & \beta_{c} & \gamma_{c}\end{array}\right]^{T}$ produced from guidance module which is the control system total input signals. The subscript "nn" represents the simulation results proposed by neural network robust adaptive inversion control law when aerodynamic parameter perturbation exists. " $c$ " is the initial command value. $\|V\|,\|W\|$ are the weight norm of neural network matrixes.

Figure 8 presents the simulation curves of RBV flight control based on neural network robust adaptive inversion. From the curves of guidance command tracking, the system output can track the change of system input finally. The sideslip angle and roll angle have the tracking errors during the attitude adjusting phase for $46.9 \mathrm{~s}$, but both are in the tolerance range. Besides, after accomplishing the adjusting phase at $140 \mathrm{~s}$, the thrust increasing produces some oscillations, which have great influence on angle of attack, but little to sideslip angle and roll angle. The errors of sideslip angle and roll angle are in the tolerance range, and when it is higher than $80 \mathrm{~km}$, the error of angle of attack has little influence on the final tracking effect. From above figures, it can be proved that the final tracking accuracy is high. Analyzing the weight norm and robust adaptive coefficients, the neural network adaptive law is effective and can eliminate the inverse errors. The network weight coefficients not only represent the parameter perturbation and the influence caused by parameter perturbation with varying as system adaptive change, but also represent the influence of eliminating the inverse errors which tended to be stable.

\section{Conclusions}

According to the uncertainty of RBV model, the robust adaptive inversion control strategy based on neural network is proposed in this paper. The nonlinear simulation verifies the validity to this methodology. Using Lyapunov theory proves the ultimate uniform boundedness of RBV closed loop control system. The simulation results indicate that when aerodynamic moment parameter perturbation is $30 \%$, this methodology can reduce the requirement of RBV model accuracy and improve the control system robustness during the adjusting phase and reentry phase of RBV.

\section{Conflict of Interests}

The authors declare that there is no conflict of interests regarding the publication of this paper.

\section{Acknowledgments}

This research was supported under National Natural Science Foundation of China (no. 11372080), China Postdoctoral Science Foundation (no. 20110491028), Central University Foundation of Basic Scientific Research (no. HEUCFZ1126, no. HEUCFZ1120), and Natural Science Foundation of Heilongjiang Province (no. QC2011C020). The authors thank Professor CUI Naigang and CHEN Weidong for their help. Also the authors are indebted to CHEN Yangyang, TANG Xiaoping, and ZENG Kui for their continuous help. Finally, the authors thank the reviewers for their helpful comments and insight.

\section{References}

[1] J. M. Hank, J. S. Murphy, and R. C. Mutzman, "The X-51A Scramjet Engine flight demonstration program," in Proceedings of the 15th AIAA International Space Planes and Hypersonic Systems and Technologies Conference, Dayton, Ohio, USA, May 2008.

[2] J. G. Wallace, J. Bradford, A. C. Charania, W. J. D. Escher, and D. Eklund, "Concept study of an ARES hybrid-OS launch system," in Proceedings of the 14th AIAA/AHI International Space Planes 
and Hypersonics Systems Technologies Conference, pp. 14771494, November 2006.

[3] J. Kauffmann, "Future European launch systems in the FLPP overview and objectives," in Proceedings of the 57th AIAA International Astronautical Congress (IAC '06), pp. 7618-7623, Valencia, Spain, October 2006.

[4] C. Tomatis, L. Bouaziz, T. Franck, and J. Kauffmann, "RLV candidates for European Future Launchers Preparatory Programme," Acta Astronautica, vol. 65, no. 1-2, pp. 40-46, 2009.

[5] M. Sippel, C. Manfletti, and H. Burkhardt, "Long-term/strategic scenario for reusable booster stages," Acta Astronautica, vol. 58, no. 4, pp. 209-221, 2006.

[6] M. Sippel, U. Atanassov, J. Klevanski, and V. Schmid, "Firststage design variations of partially reusable launch vehicles," Journal of Spacecraft and Rockets, vol. 39, no. 4, pp. 571-579, 2002.

[7] Y. Shtessel, C. Tournes, and D. Krupp, "Reusable launch vehicle control in sliding modes," in Proceedings of the AIAA Guidance, Navigation, and Control Conference, pp. 2915-2940, New Orleans, La, USA, August 2005.

[8] Y. Shtessel, J. McDuffie, M. Jackson et al., "Sliding mode control of the X-33 vehicle in launch and re-entry modes," in Proceedings of the AIAA Guidance, Navigation, and Control Conference and Exhibit, Boston, Mass, USA, 1998.

[9] Y. Shtessel, C. Hall, and M. Jackson, "Reusable launch vehicle control in multiple time scale sliding modes," in Proceedings of the AIAA Guidance, Navigation, and Control Conference and Exhibit, Denver, Colo, USA, August 2000.

[10] L. Zhang and P. Shi, " $l_{2}-l_{\infty}$ model reduction for switched LPV systems with average dwell time," IEEE Transactions on Automatic Control, vol. 53, no. 10, pp. 2443-2448, 2008.

[11] L. Zhang and P. Shi, "Stability, $l_{2}$-gain and asynchronous $H_{\infty}$ control of discrete-time switched systems with average dwell time," IEEE Transactions on Automatic Control, vol. 54, no. 9, pp. 2192-2199, 2009.

[12] L. Zhang, P. Shi, E.-K. Boukas, and C. Wang, " $H_{\infty}$ model reduction for uncertain switched linear discrete-time systems," Automatica, vol. 44, no. 11, pp. 2944-2949, 2008.

[13] Ndres, K. Murry, D. Z. Gabriele, F. P. Luis et al., "Application of LPV/LFT modeling and data-based validation to a re-entry vehicle," in Proceedings of the AIAA Guidance, Navigation, and Control Conference, Chicago, Ill, USA, August 2009.

[14] P. P. Menon, E. Prempain, I. Postlethwaite, D. Bates, and S. Bennani, "An LPV loop shaping controller design for the NASAHL-20 re-entry vehicle," in Proceedings of the AIAA Guidance, Navigation, and Control Conference and Exhibit, Chicago, Ill, USA, August 2009.

[15] P. P. Menon, E. Prempain, I. Postlethwaite, D. Bates, and S. Bennani, "Nonlinear worst-case analysis of an LPV controller for approach-phase of a re-entry vehicle," in Proceedings of the AIAA Guidance, Navigation, and Control Conference and Exhibit, August 2009.

[16] Snell, D. F. Enns, and W. L. Garrard, "Nonlinear inversion flight control for a super maneuverable aircraft," in Proceedings of the AIAA Guidance, Navigation and Control Conference, Portland, Ore, USA, 1990.

[17] D. Bugajski and D. Enns, "Nonlinear control law with application to high angle-of-attack flight," Journal of Guidance, Control, and Dynamics, vol. 15, no. 3, pp. 761-767, 1992.

[18] M. B. Farland and C. N. D’Souza, "Missile flight control with dynamic inversion and structured singular value synthesis," in Proceedings of the AIAA Guidance, Navigation, and Control Conference, pp. 544-550, Washington, DC, USA, 1994.
[19] L. H. Carter and J. S. Shamma, "Gain-scheduled bank-to-turn autopilot design using linear parameter varying transformations," Journal of Guidance, Control, and Dynamics, vol. 19, no. 5, pp. 1056-1063, 1996.

[20] J. J. Burken, P. Lu, and Z. Wu, "Reconfigurable flight control designs with application to the X-33 vehicle," in Proceedings of the Guidance, Navigation, and Control Conference, Portland, Ore, USA, 1999.

[21] Y. Minami, S. Ishimoto, T. Mori, and K. Fujii, "Design study on a small-sized partially reusable launch system," in Proceedings of the 13th AIAA/CIRA International Space Planes and Hypersonic Systems and Technologies Conference, pp. 471-482, May 2005.

[22] K. Fujii and S. Ishimoto, "Research activities to realize advanced space transportation system," in Proceedings of the 15th AIAA International Space Planes and Hypersonic Systems and Technologies Conference, May 2008.

[23] N. Goto, T. Kawakita, and J. Kawaguchi, "Nonlinear dynamical analysis for a reentry vehicle," Transactions of the Japan Society for Aeronautical and Space Sciences, vol. 47, no. 156, pp. 99-107, 2004.

[24] P. R. Jia, K. J. Chen, and L. He, Ballistics of Long Range Missiles, National University of Defense Technology Press, Changsha, China, 1993.

[25] Q. P. Ha, Q. H. Nguyen, D. C. Rye, and H. F. Durrant-Whyte, "Fuzzy sliding-mode controllers with applications," IEEE Transactions on Industrial Electronics, vol. 48, no. 1, pp. 38-46, 2001.

[26] J. Alise and N. Eric, "Reusable launch vehicle adaptive guidance and control using neural networks," in Proceedings of the AIAA Guidance, Navigation, and Control Conference, Montreal, Canada, August 2001.

[27] L. Zhang, H. Gao, and O. Kaynak, "Network-induced contraints in networked control system: a survey," IEEE Transcations on Industrial Informatics, vol. 9, no. 1, pp. 403-416, 2013.

[28] D. Bugajski and D. Enns, "Nonlinear control law with application to high angle-of-attack flight," Journal of Guidance, Control, and Dynamics, vol. 15, no. 3, pp. 761-767, 1992.

[29] F. Enns, D. J. Bugajski, R. Hendrick et al., "Dynamic inversion: an evolving methodology for flight control," International Journal of Control, vol. 59, no. 1, pp. 71-91, 1994.

[30] R. R. Da Costa, Q. P. Chu, and J. A. Mulder, "Reentry flight controller design using nonlinear dynamic inversion," Journal of Spacecraft and Rockets, vol. 40, no. 1, pp. 64-71, 2003.

[31] X. Jiangtao, Research on Attitude Control and Control Allocation for Reusable Boosted Vehicle, School of Aeronautics, Harbin Institute of Technology, Harbin, China, 2010, Chinese.

[32] McFarland and A. J. Calise, "Multilayer neural networks and adaptive control of agile anti-air missile," in Proceedings of the AIAA Guidance, Navigation, and Control Conference, New Orleans, La, USA, 1997.

[33] M. M. Polycarpou and P. A. Ioannou, "Modelling, identification and stable adaptive control of continuous-time nonlinear dynamical systems using neural networks," in Proceedings of the American Control Conference, pp. 36-40, June 1992.

[34] M. M. Polycarpou and M. J. Mears, "Stable adaptive tracking of uncertain systems using nonlinearly parametrized on-line approximators," International Journal of Control, vol. 70, no. 3, pp. 363-384, 1998.

[35] K. Madyastha, Adaptive estimation for control of uncertain nonlinear system with applications to target tracking [Ph.D. dessertation], Georgia Institute of Technology, Atlanta, Ga, USA, 2005. 


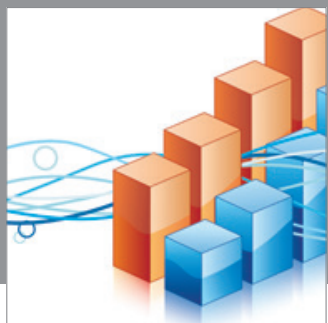

Advances in

Operations Research

mansans

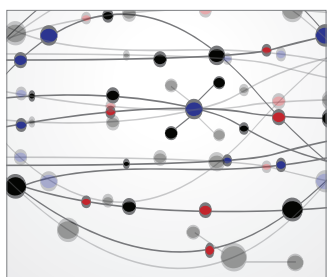

The Scientific World Journal
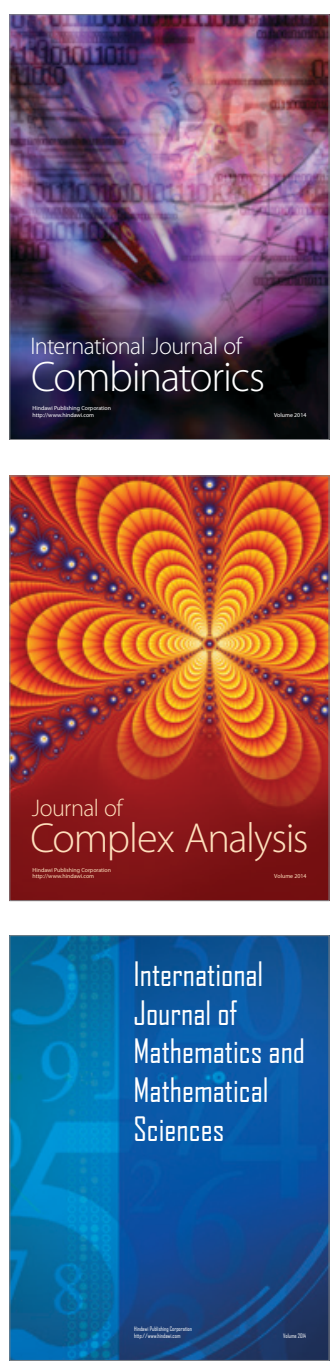
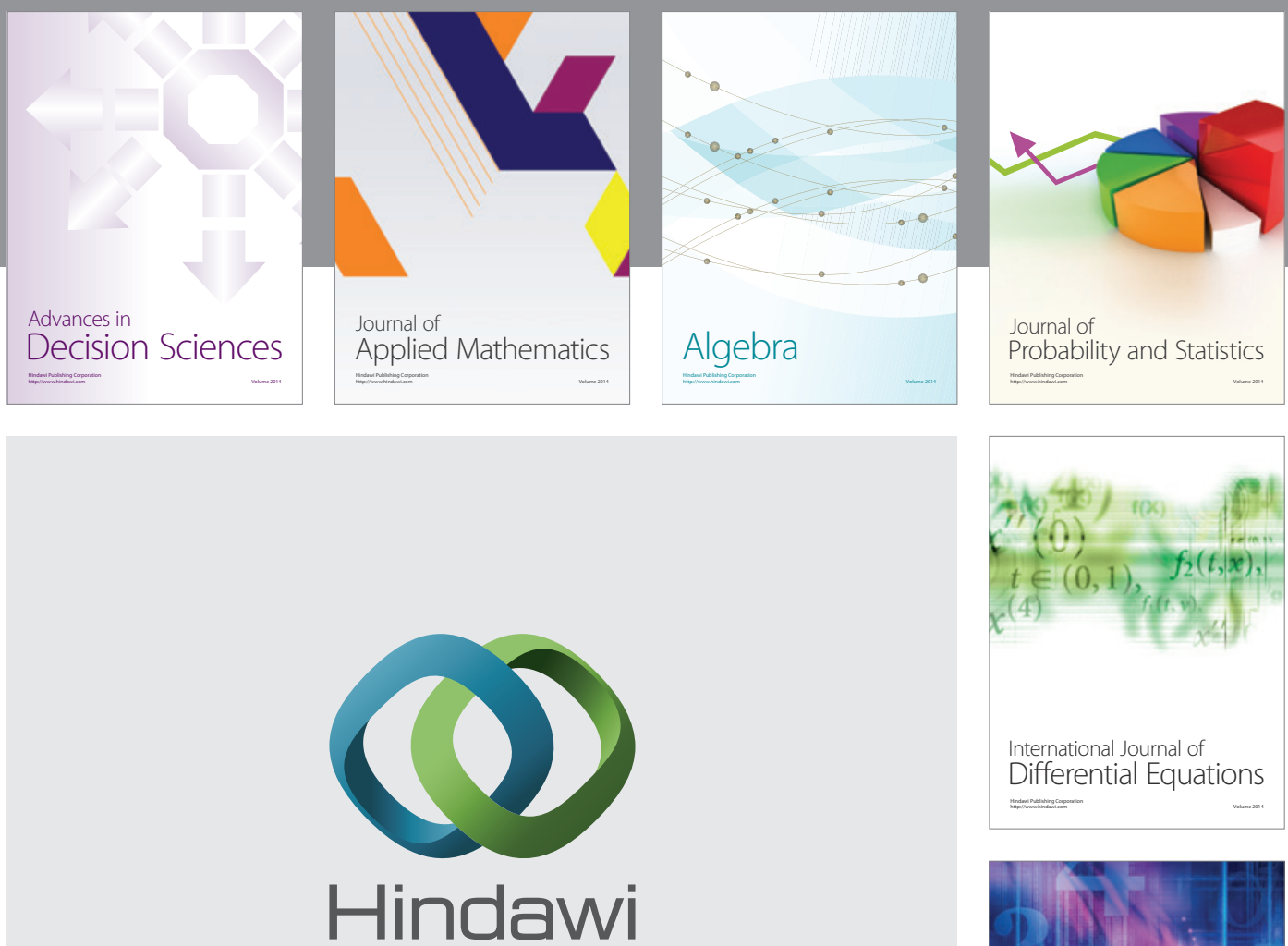

Submit your manuscripts at http://www.hindawi.com
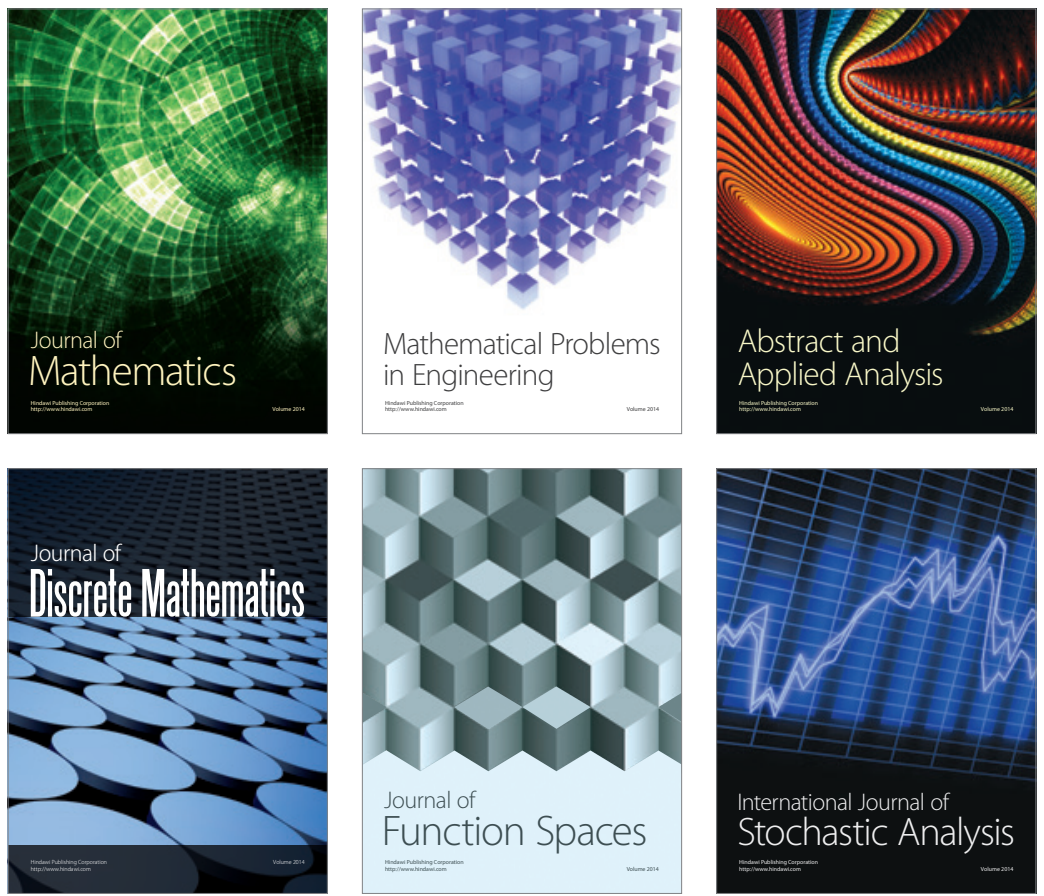

Journal of

Function Spaces

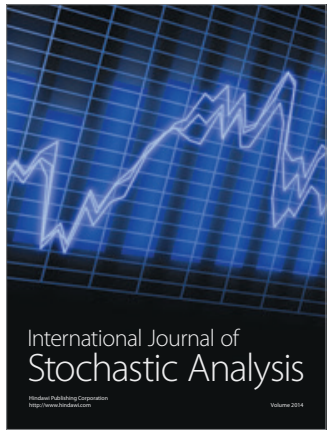

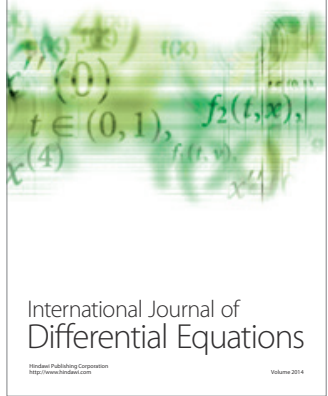
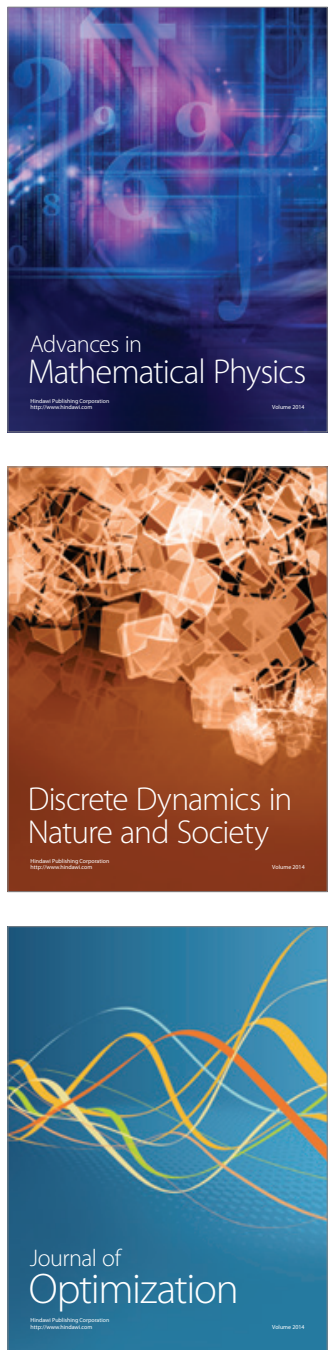